\title{
ORIGINAL ARTICLE \\ CONVENTIONAL ECHOCARDIOGRAPHY, AORTIC ELASTICITY AND LIPID PROFILES IN OBESE VERSUS HEALTHY CHILDREN
}

\author{
Noor Mohammad Noori, Maryam Nakhaee Moghadam, Alireza Teimouri ${ }^{1}$ \\ ${ }^{1}$ Children \& Adolescent Health Research Center, Resistant Tuberculosis Institute, School of Medicine, Zahedan Univ Med Sci, Zahedan, \\ Iran
}

Objectives: To compare conventional echocardiography, aortic elasticity and lipid profiles in overweight or obese children with healthy controls.

Methodology: This case control study was conducted on 49 obese or overweight children aged 4 to 16 years, equally matched in age and sex of control that were normal regarding body mass index. Echocardiography and aortic elasticity findings measured for both groups and lipid profiles measured for overweight or obese children only. Data analyzed by SPSS 20 considering 0.05 as significant level.

Results: Most of the echocardiography findings such as $\operatorname{LVDS}(\mathrm{P}<0.001), \operatorname{LVDD}(\mathrm{P}<0.001)$, $\mathrm{PWD}(\mathrm{P}<0.001)$, IVSS $(\mathrm{P}<0.001)$, LVMI $(\mathrm{P}<0.001), \mathrm{AS}(\mathrm{P}<0.001), \mathrm{AD}(\mathrm{P}<0.001)$ were higher significantly in obese children whereas, $\mathrm{FS}(\mathrm{P}<0.001), \mathrm{FS}(\mathrm{P}<0.001)$, AS beta index $(\mathrm{P}<0.001)$ and PSEM $(\mathrm{P}<0.001)$ were lower compared to healthy. PWD was higher in obese $(0.51 \pm 0.08 \mathrm{vs}$ $0.46 \pm 0.07)$ compared to healthy children. Among obese or overweight children, aortic diameter in systole $(\mathrm{AoS})(\mathrm{p}=0.025)$ was higher in those with high triglyceride level. LVMI changed from those who had LDL >130. Those who had abnormal LDL (>130) had lower value of AOS $(\mathrm{p}=0.017)$. Systolic BP was correlated with AD $(\mathrm{p}=0.007)$, Diastolic BP with AS beta Index $(\mathrm{p}=0.006)$, AoD with AS $(\mathrm{p}=0.002)$, with AD $(\mathrm{p}<0.001)$, with AS beta Index $(\mathrm{p}=0.001)$ and with PSEM $(\mathrm{P}<0.001)$

Conclusion: Heart functions were most at risk in obese children. Amongst obese or overweight children, PWD was higher, when all other heart function were similar. LVMI was higher in children with elevated LDL level.

Keywords: conventional echocardiography, aortic elasticity, lipid profiles, obese children

Citation: Noori NM, Moghadam MN, Teimouri A. Conventional Echocardiography, Aortic Elasticity and Lipid
Profiles in Obese Versus Healthy Children. Pak Heart J. 2021;54(02):172-179.
https://doi.org/10.47144/phj.v54i2.2095

\section{INTRODUCTION}

One of the most important public health problems is obesity with an increasing rate and variation of prevalence in different societies. ${ }^{1}$ Has been reported a very high prevalence affected by sociocultural and environmental factors in which controls with diet and physical activities. ${ }^{1}$ An increasing obesity rate occurred in Iranian children population with a slow trend. $^{2}$ Obesity has a close connection with dyslipidemia, heart disease, diabetes, malignancies and short life expectancy ${ }^{1-3}$ such that if they take long time in children, the obesity would have a great effect on cardiovascular diseases (CVD) and atherosclerosis even in adulthood. ${ }^{3}$ Obese population had higher death rate than general population due to different acute diseases with a similar trend in pediatric population. ${ }^{4}$ During regular clinical care, the early CVD are not treated due to lack of accurate diagnosis in obese children. ${ }^{3}$ Therefore, it is necessary to detect on time CVD and starting the treatment in obese children to control cardiac abnormalities in adulthood. ${ }^{5}$ Since CVD is a main cause of death around the world, makes us to improve our awareness and knowledge about the risk of CVD during childhood. One of these risk factors that most of the population doesn't know is arterial stiffening. ${ }^{6}$ Therefore, blood vessel stiffening outlined as a brand new detectable manifestation of adverse structural and functional changes within the vessel wall that reflects gradual fragmentation and loss of elastin fibers and accumulation of stiffer collagen fibers within the blood vessel wall. ${ }^{7}$ Stiffening of the arteries may be a physiological consequence as a result of aging. ${ }^{3}$ In addition, different factors like dyslipidemia could accelerate this complication. ${ }^{8}$ It's conjointly been instructed that stiffing is influenced by gender and time of life, caused by variations in sex steroids. ${ }^{3}$ Ethnicity may additionally influence blood vessel stiffening, since it's been shown that South-Asians and Africans have higher blood vessel stiffening as compared with Caucasians. ${ }^{9}$ Expressed that at least two decades living with obesity is an independent risk factor CVD and a-10 kg gain in weight increases the risk of CVD by $12 \%$ and, systolic and diastolic blood pressures increased to $3 \mathrm{mmHg}$ $2.3 \mathrm{mmHg}$ in the order given. ${ }^{10}$ Arterial stiffening is 
delineating as elastic resistance to deformation, and is full of advanced interactions between vascular smooth muscle cells and also the extracellular matrix containing albuminoidal, collagen, and fibril in fibers. ${ }^{11}$ Despite of aging, blood vessel stiffening influenced by several different diseases like celiac, asthma, diabetes, end-stage renal disease and thalassemia. ${ }^{11}$ In fact, $70 \%$ of obese children have at least one CVD risk issue, and $39 \%$ have 2 or more. CVD risk factors, together with avoirdupois, aren't related to cardiopathy in childhood but are associated with an increased prevalence of CVD risk factors in adults. ${ }^{12}$

Recently, studies on the impact of lipid profiles the level of obese children highlighted but without stiffing considerations but found arterial stiffness increased rapidly from early to late adolescence among obese and vascular dysfunction not observed among prepubertal obese. Based on the mentioned above materials, the present study aimed to assess conventional echocardiography, stiffing parameters in obese children compared with healthy and comparing these parameters in obese children based on lipid profile variations.

\section{METHODOLOGY}

The study followed a case-control style and was performed on ninety-eight kids aged 6 to 19 years equally shared in healthy and obese or overweight in pediatric cardiac center collaborated with the center for specific diseases in Ali Asghar Hospital, Zahedan, Sistan and Baluchestan province, Iran, from 2018 September to 2019 August.

The exclusion criteria for the study were as follow; thyroid hormone abnormality, nephrotic syndrome, autoimmune disease, hepatic disorders, cirrhosis, valvular disease, rhythm abnormality, infectious diseases, systemic inflammatory disorder, renal failure and sleep apnea.

The children went under checking for medical history, physical examination, chest $\mathrm{X}$-ray and echocardiography that was performed using My lab 60 with transducer 3, 8 (made in Italy). Echocardiogram performed in without breathing control status and for more precision the measurement was repeated for 3 cycles and the average was considered.

Conventional echocardiography parameters that were used in the study were: diastolic diameter of the aorta (Aod), systolic diameter of the aorta (Aos), left ventricular end diastolic dimension (LVDD), posterior wall dimension in diastole (PWD), interventricular septal dimension in Diastole (IVSD), interventricular septal dimension in systole (IVSS), relative wall thickness (RWT) that defined as 2 times PWT divided by the LV diastolic diameter, Ejection fraction (EF), fractional shortening (FS), left ventricular mass (LVM) and left ventricular mass index (LVMI) were measured using conventional echocardiography of the left side and estimated from three cardiac cycles. LVMI was calculated by the following formula:

LVM $(\mathrm{g})=0.8\left(1.04\left(\mathrm{LVDD}+\mathrm{PWD}+\mathrm{IVSD}^{3}{ }^{3}\right.\right.$ $\left.\mathrm{LVDD}^{3}\right)+0.6$, and LVMI $(\mathrm{g} / \mathrm{m} 2)=\mathrm{LVM} / 2.7$ $\left(\mathrm{g} / \mathrm{m}^{2}\right)$. These parameters measured utilized for left ventricular mass evaluation. ${ }^{13}$

After echocardiography, measuring aortic diameter obtained from $3 \mathrm{~cm}$ above the aortic valve by the $\mathrm{M}$ mode. Aortic diameters were calculated as the distance between the anterior and posterior wall inner edges of the aorta at systole and diastole. AoS was recorded when the aortic wall was fully open. AoD was recorded simultaneously when the QRS peak was seen on electrocardiographic (ECG) recordings. Measurements were taken during three consecutive pulses and the mean was calculated.

Blood pressure (BP) were measured from the brachial artery with a sphygmomanometer after at least 5 minutes resting in the supine position. Three measurements, at least 2 minutes apart applied and the average of the closest two readings recorded. A pressure drop rate of approximately $2 \mathrm{~mm} \mathrm{Hg} / \mathrm{s}$ applied, and Korotkoff phases I and V used for systolic and diastolic BP respectively.

The ascending aortic diameters recorded in M-mode approximately $3 \mathrm{~cm}$ above the aortic valve from parasternal long axis views. The systolic aortic diameter measured at the time of maximum anterior motion of the aorta while the diastolic diameter measured at the start of the QRS complex in electrocardiography (Figure 1). Aortic elasticity parameters calculated as follow. ${ }^{11}$

Aortic strain $(\%)=($ aortic SD - aortic DD) $\times 100$ /aortic DD

Aortic stiffness beta index = natural logarithm (systolic BP/diastolic BP)/ ([aortic SD-aortic DD]/aortic DD)

Aortic distensability $\left(\mathrm{cm}^{2} \cdot\right.$ dyne-1.10-6) $=2 x$ ([aortic SD-aortic DD]/ aortic DD) / (SBP-DBP)

Pressure strain elastic modulus $=(\mathrm{SBP}-\mathrm{DBP}) /$ ([aortic SD-aortic DD]/aortic DD)

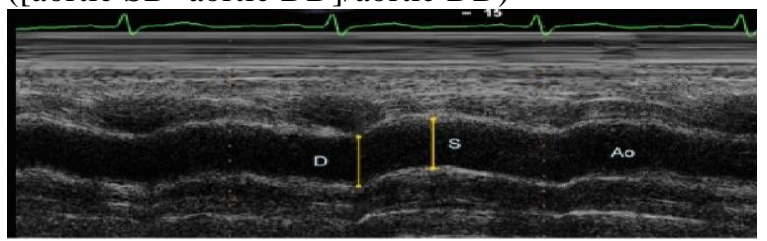

Figure 1: Measurements of systolic (S) and diastolic (D) diameters of the ascending aorta are shown on the $M$ mode tracing obtained at a level $3 \mathrm{~cm}$ above the aortic valve 
Lipid profiles of CHO mg/dl, HDL mg/dl, LDL $\mathrm{mg} / \mathrm{dl}$, and TG $\mathrm{mg} / \mathrm{dl}$ considered for the study with cut points of $\mathrm{CHO}>200 \mathrm{mg} / \mathrm{dl}$, HDL $<40 \mathrm{mg} / \mathrm{dl}$, $\mathrm{LDL}>130 \mathrm{mg} / \mathrm{dl}$, and $\mathrm{TG}>150 \mathrm{mg} / \mathrm{dl}$ as abnormal levels. $^{14}$

Anthropomorphic measurements like height and weight were measured for the participants by an experienced nurse with standard equipment. Height measured in the standing position with a balance using a scaled ruler and weight measured using a RASA scale factor with an error of $100 \mathrm{~g}$ (made in Iran). BMI calculated as weight $(\mathrm{kg}) /$ height $^{2}\left(\mathrm{~m}^{2}\right)$. From the participants or their guardians consent was taken after the study approval. The study was approved as a project proposed to the Children and Adolescent Health Research Center by the Ethics Committee of Zahedan University of Medical Sciences, Zahedan, Iran (IR.ZAUMS.REC.1400.095).

Data were analyzed using SPSS for Windows version 20.0 (SPSS Inc., Chicago, IL, USA). Kolmogorov-Smirnov test applied to assess distribution of continuous variables where homogeneity was tested. Student's t-test was used to compare mean values of normal quantitative variables while Mann-Whitney $U$ test used for the variables with skewed distribution. In correlation analyses, Pearson chi squire test was used for parametric variables. $P$ value $\leq 0.05$ was considered as statistically significant.

\section{RESULTS}

The present study aimed to assess conventional echocardiography, stiffing parameters and lipid profiles in obese children. The normality test showed height $(\mathrm{p}=0.053)$, AoS $(\mathrm{p}=0.062), \mathrm{LVDD}$ $(\mathrm{p}=0.200)$, LVDS $(\mathrm{p}=0.165)$, EF $(\mathrm{p}=0.200)$, AS $(\mathrm{p}=0.200), A D(\mathrm{p}=0.184)$ and LVMI $(\mathrm{p}=0.061)$ had normal distribution in obese and AoS $(\mathrm{p}=0.220)$ and LVDS $(\mathrm{p}=0.093)$ were normal in all participants.

Gender distribution was similar ( $\mathrm{X} 2=1.048$ and $\mathrm{p}=0.306$ ) in gender group of participants such that obese girls and boys were $43.9 \%$ and $54.4 \%$ respectively. Height $(\mathrm{p}<0.001)$, SBP $(\mathrm{p}=0.002)$, AoS $(\mathrm{p}<0.001)$, AoD $(\mathrm{p}=0.002)$, LVDS $(\mathrm{p}<0.001)$, LVDD $\quad(p<0.001), \quad$ PWD $\quad(p<0.001), \quad$ IVSS $(p<0.001), \quad$ LVDS $\quad(p<0.001), \quad$ PWS $\quad(p<0.001)$, LVMI $(\mathrm{p}<0.001)$ AS $(\mathrm{p}<0.001)$, AD $(\mathrm{p}<0.001)$ were higher in obese children significantly whereas FS $(\mathrm{p}<0.001)$, FS $(\mathrm{p}<0.001)$, AS beta index $(\mathrm{p}<0.001)$ and PSEM $(\mathrm{p}<0.001)$ were lower compared to children with normal BMI. The parameter of PWD was different between obese and overweight children in favor of obese children $(0.51 \pm 0.08$ vs. $0.46 \pm 0.07)$ (Table1). Lipid profiles of CHO $>200 \mathrm{mg} / \mathrm{dl}(2.25 \mathrm{mmol} / \mathrm{l}) \quad$ and TG >>150(1.69 mmol/l) were abnormal and based on this issue, all heart variables were similar in obese and overweight children based on the cut off 200 and 150 as cut points for $\mathrm{CHO}$ and TG respectively except the parameter of AOS $(\mathrm{p}=0.025)$ that was higher in children with abnormal TG (Table 2). Table 3 showed that HDL $<40 \mathrm{mg} / \mathrm{dl}(0.45$ $\mathrm{mg} / \mathrm{l})$ and $\mathrm{LDL}>130(1.46 \mathrm{mmol} /)$ were abnormal such that all heart variables were similar in obese children based on the cut off 40 for HDL. Considering the LDL, only LVMI changed from those who had LDL >130 compared with those had less. Those who had abnormal LDL (>130) had lower value of AOS $(\mathrm{p}=0.017)$. Table 4 showed the correlation of aortic stiffness parameters with conventional echocardiography parameters and lipid profiles. From the tables observed that SBP was correlated with $\mathrm{AD}(\mathrm{p}=0.007)$, $\mathrm{DBP}$ was correlated with AS beta index ( $\mathrm{p}=0.006)$, AoD was correlated with AS $(\mathrm{p}=0.002)$, AD $(\mathrm{p}<0.001)$, AS beta index $(\mathrm{p}=0.001)$ and PSEM $(\mathrm{P}<0.001)$.

Table 1: Anthropometric, echocardiography and stiffing measures comparison in Overweight and Obese children

\begin{tabular}{|c|c|c|c|c|c|c|c|c|c|c|}
\hline Variables & Groups of patients & Mean & SD & $\begin{array}{c}\text { Test } \\
\text { Value }\end{array}$ & $\mathbf{P}$ & Variables & Mean & SD & $\begin{array}{c}\text { Critical } \\
\text { Value }\end{array}$ & $\mathbf{P}$ \\
\hline \multirow{2}{*}{ Age } & Overweight & 10.19 & 2.73 & \multirow{2}{*}{-0.52} & \multirow{2}{*}{0.608} & \multirow{2}{*}{ PWS } & 0.47 & 0.08 & \multirow{2}{*}{205.5} & \multirow{2}{*}{0.056} \\
\hline & Obese & 10.56 & 2.31 & & & & 0.51 & 0.08 & & \\
\hline \multirow{2}{*}{ Weight } & Overweight & 56.33 & 12.27 & \multirow{2}{*}{172} & \multirow{2}{*}{0.01} & \multirow{2}{*}{$\mathrm{EF}$} & 0.69 & 0.06 & \multirow{2}{*}{0.12} & \multirow{2}{*}{0.902} \\
\hline & Obese & 72.08 & 20.29 & & & & 0.69 & 0.06 & & \\
\hline \multirow{2}{*}{ Height } & Overweight & 142.92 & 15.86 & \multirow{2}{*}{-0.21} & \multirow{2}{*}{0.833} & \multirow{2}{*}{ FS } & 0.36 & 0.04 & \multirow{2}{*}{287.5} & \multirow{2}{*}{0.801} \\
\hline & Obese & 143.88 & 15.86 & & & & 0.36 & 0.05 & & \\
\hline \multirow{2}{*}{ AoS } & Overweight & 2.41 & 0.3 & \multirow{2}{*}{-1.21} & \multirow{2}{*}{0.232} & \multirow{2}{*}{ AS } & 18.59 & 8.08 & \multirow{2}{*}{-1.1} & \multirow{2}{*}{0.276} \\
\hline & Obese & 2.51 & 0.3 & & & & 20.7 & 5.04 & & \\
\hline \multirow{2}{*}{ AoD } & Overweight & 2.04 & 0.29 & \multirow{2}{*}{260.5} & \multirow{2}{*}{0.429} & \multirow{2}{*}{$\mathrm{AD}$} & 0.01 & 0 & \multirow{2}{*}{-1.13} & \multirow{2}{*}{0.264} \\
\hline & Obese & 2.09 & 0.3 & & & & 0.01 & 0 & & \\
\hline \multirow{2}{*}{ Systolic BP } & Overweight & 111.71 & 11.74 & \multirow{2}{*}{294.5} & \multirow{2}{*}{0.909} & \multirow{2}{*}{ AS beta Index } & 3.48 & 2.06 & \multirow{2}{*}{242} & \multirow{2}{*}{0.246} \\
\hline & Obese & 113.08 & 14.55 & & & & 2.67 & 1.15 & & \\
\hline Diastolic & Overweight & 66.83 & 5.66 & 2085 & 0075 & PSFI & 3.01 & 1.66 & 241 & 0238 \\
\hline BP & Obese & 68.4 & 11.63 & 290.5 & 0.913 & FSEIVI & 2.34 & 1.01 & 241 & 0.230 \\
\hline
\end{tabular}




\begin{tabular}{|c|c|c|c|c|c|c|c|c|c|c|}
\hline \multirow{2}{*}{ IVSD } & Overweight & 1.01 & 0.19 & \multirow{2}{*}{291.5} & \multirow{2}{*}{0.864} & \multirow{2}{*}{ LVMI } & 55.68 & 17.08 & \multirow{2}{*}{-1.46} & \multirow{2}{*}{0.151} \\
\hline & Obese & 1.02 & 0.18 & & & & 63.79 & 21.51 & & \\
\hline \multirow{2}{*}{ LVDD } & Overweight & 4.23 & 0.52 & \multirow{2}{*}{-1.3} & \multirow{2}{*}{0.201} & \multirow{2}{*}{ CHOLESTROL } & 178.96 & 32.26 & \multirow{2}{*}{279.5} & \multirow{2}{*}{0.682} \\
\hline & Obese & 4.43 & 0.55 & & & & 173.56 & 27.22 & & \\
\hline \multirow{2}{*}{ PWD } & Overweight & 0.46 & 0.07 & \multirow{2}{*}{191.5} & \multirow{2}{*}{0.028} & \multirow{2}{*}{ HDL } & 51.92 & 22.8 & \multirow{2}{*}{298} & \multirow{2}{*}{0.968} \\
\hline & Obese & 0.51 & 0.08 & & & & 51.4 & 22.1 & & \\
\hline \multirow{2}{*}{ IVSS } & Overweight & 1.15 & 0.16 & \multirow{2}{*}{246} & \multirow{2}{*}{0.275} & \multirow{2}{*}{ LDL } & 99.75 & 24.68 & \multirow{2}{*}{299} & \multirow{2}{*}{0.984} \\
\hline & Obese & 1.22 & 0.2 & & & & 98.52 & 20.92 & & \\
\hline \multirow{2}{*}{ LVDS } & Overweight & 2.57 & 0.39 & \multirow{2}{*}{-1.08} & \multirow{2}{*}{0.285} & \multirow{2}{*}{ TG } & 139.38 & 52.54 & \multirow{2}{*}{265} & \multirow{2}{*}{0.484} \\
\hline & Obese & 2.7 & 0.46 & & & & 130.64 & 53.24 & & \\
\hline
\end{tabular}

Table 2: Conventional echocardiography and aortic stiffing parameters changes in overweight and obese children based on $\mathrm{CHO}$ and TG changes $\geq$

\begin{tabular}{|c|c|c|c|c|c|c|c|c|c|c|}
\hline \multirow{2}{*}{ Variables } & \multicolumn{5}{|c|}{ CHO(mg/dl) } & \multicolumn{5}{|c|}{ TG(mg/dl) } \\
\hline & Groups & Mean & SD & Test Value & P value & Groups & Mean & SD & Test Value & P value \\
\hline \multirow{2}{*}{ IVSD } & $<200$ & 1.01 & 0.17 & \multirow{2}{*}{192.5} & \multirow{2}{*}{0.951} & $<150$ & 1.01 & 0.18 & \multirow{2}{*}{231} & \multirow{2}{*}{0.756} \\
\hline & $\geq 200$ & 1.02 & 0.22 & & & $\geq 150$ & 1.03 & 0.19 & & \\
\hline \multirow{2}{*}{ LVDD } & $<200$ & 4.35 & 0.57 & \multirow{2}{*}{186.5} & \multirow{2}{*}{0.836} & $<150$ & 4.29 & 0.57 & \multirow{2}{*}{182.5} & \multirow{2}{*}{0.166} \\
\hline & $\geq 200$ & 4.26 & 0.41 & & & $\geq 150$ & 4.43 & 0.44 & & \\
\hline \multirow{2}{*}{ IVSS } & $<200$ & 1.18 & 0.19 & \multirow{2}{*}{162.5} & \multirow{2}{*}{0.426} & $<150$ & 1.18 & 0.19 & \multirow{2}{*}{226} & \multirow{2}{*}{0.671} \\
\hline & $\geq 200$ & 1.21 & 0.16 & & & $\geq 150$ & 1.19 & 0.16 & & \\
\hline \multirow{2}{*}{ LVDS } & $<200$ & 2.66 & 0.44 & \multirow{2}{*}{0.794} & \multirow{2}{*}{0.431} & $<150$ & 2.58 & 0.43 & \multirow{2}{*}{-1.428} & \multirow{2}{*}{0.16} \\
\hline & $\geq 200$ & 2.54 & 0.36 & & & $\geq 150$ & 2.77 & 0.41 & & \\
\hline \multirow{2}{*}{ PW } & $<200$ & 0.49 & 0.08 & \multirow{2}{*}{186.5} & \multirow{2}{*}{0.836} & $<150$ & 0.49 & 0.08 & \multirow{2}{*}{244} & 0.082 \\
\hline & $\geq 200$ & 0.5 & 0.09 & & & $\geq 150$ & 0.49 & 0.08 & & 0.982 \\
\hline $\mathrm{FE}$ & $<200$ & 0.69 & 0.06 & 0757 & 0453 & $<150$ & 0.7 & 0.06 & 1182 & 0243 \\
\hline $\mathrm{EF}$ & $\geq 200$ & 0.71 & 0.05 & $-0.15 /$ & 0.453 & $\geq 150$ & 0.68 & 0.06 & 1.182 & 0.243 \\
\hline$\Gamma \mathrm{C}$ & $<200$ & 0.35 & 0.04 & 140 & (2002 & $<150$ & 0.36 & 0.04 & 210 & $0=126$ \\
\hline FS & $\geq 200$ & 0.38 & 0.05 & 148 & 0.253 & $\geq 150$ & 0.36 & 0.06 & 210 & 0.436 \\
\hline$\Delta S_{-}$ & $<200$ & 19.29 & 6.76 & 0767 & 0447 & $<150$ & 19.76 & 6.95 & 0154 & 0878 \\
\hline AS & $\geq 200$ & 21.13 & 6.72 & -0.767 & 0.447 & $\geq 150$ & 19.43 & 6.36 & 0.154 & 0.878 \\
\hline & $<200$ & 0.01 & 0 & & & $<150$ & 0.01 & 0 & & \\
\hline AD & $\geq 200$ & 0.01 & 0 & -0.737 & 0.465 & $\geq 150$ & 0.01 & 0 & 0.442 & 0.66 \\
\hline$\Delta \mathrm{S}$ heto Ind & $<200$ & 3.2 & 1.78 & & & $<150$ & 3.1 & 1.8 & 243 & 0065 \\
\hline AS beta Index & $\geq 200$ & 2.53 & 1.2 & 150 & 0.274 & $\geq 150$ & 2.98 & 1.44 & 243 & 0.965 \\
\hline PSFM & $<200$ & 2.78 & 1.47 & 155 & 0333 & $<150$ & 2.66 & 1.47 & 220 & 0723 \\
\hline PSEM & $\geq 200$ & 2.2 & 0.98 & 155 & 0.333 & $\geq 150$ & 2.69 & 1.22 & 229 & 0.723 \\
\hline Systolic BP & $<200$ & 113.1 & 13.07 & 168 & 0.517 & $<150$ & 110.71 & 12.23 & 178 & 0.124 \\
\hline Systolic BP & $\geq 200$ & 109.7 & 13.7 & 168 & 0.517 & $\geq 150$ & 116.64 & 14.77 & $1 / 8$ & 0.124 \\
\hline Diostalis PD & $<200$ & 67.49 & 9.39 & 186 & 0.836 & $<150$ & 66.49 & 8.6 & 1065 & 027 \\
\hline Diastolic BP & $\geq 200$ & 68.2 & 8.53 & 186 & 0.836 & $\geq 150$ & 70.5 & 10.14 & 196.5 & 0.27 \\
\hline AOS & $<200$ & 24.67 & 3.27 & 0364 & & $<150$ & 23.97 & 2.73 & 2321 & \\
\hline AOS & $\geq 200$ & 24.27 & 2.06 & 0.364 & 0.717 & $\geq 150$ & 26.11 & 3.34 & -2.321 & 0.025 \\
\hline AOD & $<200$ & 20.75 & 3.15 & 1605 & 0.523 & $<150$ & 20.09 & 2.72 & 1575 & 0052 \\
\hline$A O D$ & $\geq 200$ & 20.09 & 2 & 169.5 & 0.533 & $\geq 150$ & 21.93 & 3.18 & $15 \% .5$ & 0.053 \\
\hline & $<200$ & 60.25 & 20.84 & & & $<150$ & 58.7 & 21.32 & & \\
\hline LVMI & $\geq 200$ & 58.16 & 15.17 & 0.296 & 0.768 & $\geq 150$ & 62.62 & 15.23 & -0.626 & 0.534 \\
\hline
\end{tabular}

Table 3: Conventional echocardiography and aortic stiffing parameters changes in overweight and obese children based on HDL and LDL changes

\begin{tabular}{|c|c|c|c|c|c|c|c|c|c|c|c|c|}
\hline \multirow[b]{2}{*}{ Variables } & \multicolumn{6}{|c|}{ HDL(mg/dl) } & \multicolumn{6}{|c|}{ LDL(mg/dl) } \\
\hline & Groups & $\mathbf{N}$ & Mean & SD & $\begin{array}{c}\text { Test } \\
\text { Value }\end{array}$ & $\begin{array}{c}P \\
\text { value }\end{array}$ & Groups & $\mathbf{N}$ & Mean & SD & $\begin{array}{c}\text { Test } \\
\text { Value }\end{array}$ & $P$ value \\
\hline \multirow[b]{2}{*}{ IVSD } & $<40$ & 7 & 1.03 & 0.18 & \multirow[b]{2}{*}{137} & \multirow[b]{2}{*}{0.774} & $<130$ & 46 & 1.02 & 0.18 & \multirow[b]{2}{*}{38.00} & \multirow[b]{2}{*}{0.194} \\
\hline & $\geq 40$ & $\begin{array}{l}4 \\
2\end{array}$ & 1.01 & 0.18 & & & $\geq 130$ & 3 & 0.89 & 0.06 & & \\
\hline \multirow[b]{2}{*}{ LVDD } & $<40$ & 7 & 4.57 & 0.52 & \multirow[b]{2}{*}{105} & \multirow[b]{2}{*}{0.235} & $<130$ & 46 & 4.32 & 0.55 & \multirow[b]{2}{*}{54.00} & \multirow[b]{2}{*}{0.531} \\
\hline & $\geq 40$ & $\begin{array}{l}4 \\
2\end{array}$ & 4.29 & 0.54 & & & $\geq 130$ & 3 & 4.45 & 0.25 & & \\
\hline \multirow[b]{2}{*}{ IVSS } & $<40$ & 7 & 1.18 & 0.16 & \multirow[b]{2}{*}{145.5} & \multirow[b]{2}{*}{0.965} & $<130$ & 46 & 1.19 & 0.19 & \multirow[b]{2}{*}{47.00} & \multirow[b]{2}{*}{0.354} \\
\hline & $\geq 40$ & $\begin{array}{l}4 \\
2\end{array}$ & 1.19 & 0.19 & & & $\geq 130$ & 3 & 1.09 & 0.11 & & \\
\hline LVDS & $<40$ & 7 & 2.86 & 0.37 & 1.52 & 0.135 & $<130$ & 46 & 2.64 & 0.44 & 0.53 & 0.602 \\
\hline
\end{tabular}




\begin{tabular}{|c|c|c|c|c|c|c|c|c|c|c|c|c|}
\hline & $\geq 40$ & $\begin{array}{l}4 \\
2\end{array}$ & 2.60 & 0.43 & & & $\geq 130$ & 3 & 2.51 & 0.24 & & \\
\hline \multirow[b]{2}{*}{ PW } & $<40$ & 7 & 0.46 & 0.06 & \multirow[b]{2}{*}{105} & \multirow[b]{2}{*}{0.225} & $<130$ & 46 & 0.49 & 0.08 & \multirow[b]{2}{*}{43.00} & \multirow[b]{2}{*}{0.273} \\
\hline & $\geq 40$ & $\begin{array}{l}4 \\
2\end{array}$ & 0.50 & 0.08 & & & $\geq 130$ & 3 & 0.45 & 0.07 & & \\
\hline \multirow[b]{2}{*}{ EF } & $<40$ & 7 & 0.68 & 0.06 & \multirow[b]{2}{*}{-0.379} & \multirow[b]{2}{*}{0.706} & $<130$ & 46 & 0.69 & 0.06 & \multirow[b]{2}{*}{-0.73} & \multirow[b]{2}{*}{0.469} \\
\hline & $\geq 40$ & $\begin{array}{l}4 \\
2\end{array}$ & 0.69 & 0.06 & & & $\geq 130$ & 3 & 0.72 & 0.07 & & \\
\hline \multirow[b]{2}{*}{ FS } & $<40$ & 7 & 0.35 & 0.04 & \multirow[b]{2}{*}{143} & \multirow[b]{2}{*}{0.92} & $<130$ & 46 & 0.36 & 0.04 & \multirow[b]{2}{*}{44.00} & \multirow[b]{2}{*}{0.294} \\
\hline & $\geq 40$ & $\begin{array}{l}4 \\
2\end{array}$ & 0.36 & 0.05 & & & $\geq 130$ & 3 & 0.4 & 0.09 & & \\
\hline \multirow[b]{2}{*}{ AS } & $<40$ & 7 & 22.08 & 7.15 & \multirow[b]{2}{*}{1.026} & \multirow[b]{2}{*}{0.31} & $<130$ & 46 & 20.02 & 6.71 & \multirow[b]{2}{*}{1.48} & \multirow[b]{2}{*}{0.147} \\
\hline & $\geq 40$ & $\begin{array}{l}4 \\
2\end{array}$ & 19.27 & 6.65 & & & $\geq 130$ & 3 & 14.19 & 4.74 & & \\
\hline \multirow[b]{2}{*}{$\mathrm{AD}$} & $<40$ & 7 & 0.01 & 0.01 & \multirow[b]{2}{*}{1.029} & \multirow[b]{2}{*}{0.309} & $<130$ & 46 & 0.01 & 0 & \multirow[b]{2}{*}{0.90} & \multirow[b]{2}{*}{0.378} \\
\hline & $\geq 40$ & $\begin{array}{l}4 \\
2 \\
\end{array}$ & 0.01 & 0.00 & & & $\geq 130$ & 3 & 0.01 & 0 & & \\
\hline \multirow{2}{*}{$\begin{array}{l}\text { AS beta } \\
\text { Index }\end{array}$} & $<40$ & 7 & 2.55 & 1.74 & \multirow[b]{2}{*}{103} & \multirow[b]{2}{*}{0.209} & $<130$ & 46 & 3.02 & 1.7 & \multirow[b]{2}{*}{42.00} & \multirow[b]{2}{*}{0.26} \\
\hline & $\geq 40$ & $\begin{array}{l}4 \\
2 \\
\end{array}$ & 3.15 & 1.69 & & & $\geq 130$ & 3 & 3.75 & 1.64 & & \\
\hline & $<40$ & 7 & 2.29 & 1.50 & & & $<130$ & 46 & 2.64 & 1.41 & & \\
\hline PSEM & $\geq 40$ & $\begin{array}{l}4 \\
2 \\
\end{array}$ & 2.73 & 1.38 & 108 & 0.265 & $\geq 130$ & 3 & 3.1 & 1.23 & 46.00 & 0.337 \\
\hline Systolic BP & $<40$ & 7 & 113.81 & $\begin{array}{r}10.6 \\
8 \\
\end{array}$ & 134 & 0700 & $<130$ & 46 & 112.85 & 12.82 & 46.50 & 0.331 \\
\hline & $\geq 40$ & $\begin{array}{l}4 \\
2 \\
\end{array}$ & 112.10 & $\begin{array}{r}13.5 \\
9 \\
\end{array}$ & 154 & 0.100 & $\geq 130$ & 3 & 105.67 & 19.14 & & \\
\hline & $<40$ & 7 & 72.00 & 8.70 & & & $<130$ & 46 & 67.83 & 9.26 & & \\
\hline $\begin{array}{l}\text { Diastolic } \\
\text { BP }\end{array}$ & $\geq 40$ & $\begin{array}{l}4 \\
2\end{array}$ & 66.90 & 9.11 & 92.5 & 0.110 & $\geq 130$ & 3 & 64.67 & 8.08 & 55.50 & 0.563 \\
\hline & $<40$ & 7 & 26.27 & 3.29 & & & $<130$ & 46 & 24.67 & 3.11 & & \\
\hline AOS & $\geq 40$ & $\begin{array}{l}4 \\
2\end{array}$ & 24.30 & 2.95 & 1.607 & 0.115 & $\geq 130$ & 3 & 23.3 & 1.64 & 0.751 & 0.456 \\
\hline & $<40$ & 7 & 21.69 & 3.96 & & & $<130$ & 46 & 20.63 & 3.02 & & \\
\hline AOD & $\geq 40$ & $\begin{array}{l}4 \\
2 \\
\end{array}$ & 20.44 & 2.77 & 130.5 & 0.637 & $\geq 130$ & 3 & 20.43 & 1.72 & 66.50 & 0.917 \\
\hline & $<40$ & 7 & 64.36 & 17.7 & & & $<130$ & 46 & 60.28 & 20.25 & & \\
\hline LVMI & $\geq 40$ & $\begin{array}{l}4 \\
2 \\
\end{array}$ & 59.06 & 20.1 & 0.654 & 0.516 & $\geq 130$ & 3 & 52.74 & 1.07 & 2.473 & 0.017 \\
\hline
\end{tabular}

Table 4: Aortic stiffing correlation with echocardiography finding and lipid profiles on overweight and Obese children

\begin{tabular}{|c|c|c|c|c|c|c|c|c|c|c|c|}
\hline Variables & Statistics & AS & AD & $\begin{array}{c}\text { AS beta } \\
\text { Index }\end{array}$ & PSEM & Variables & Statistics & AS & AD & $\begin{array}{c}\text { AS beta } \\
\text { Index }\end{array}$ & PSEM \\
\hline \multirow{2}{*}{ Systolic BP } & $\mathrm{r}$ & 0.042 & -0.379 & 0.033 & 0.203 & \multirow{2}{*}{ PWS } & $\mathrm{r}$ & 0.204 & -0.129 & -0.145 & -0.108 \\
\hline & $\mathrm{p}$ value & 0.776 & 0.007 & 0.824 & 0.161 & & $\mathrm{p}$ value & 0.160 & 0.378 & 0.320 & 0.459 \\
\hline \multirow{2}{*}{$\begin{array}{l}\text { Diastolic } \\
\text { BP }\end{array}$} & $\mathrm{r}$ & 0.154 & 0.252 & -0.388 & -0.256 & \multirow{2}{*}{$\mathrm{EF}$} & $\mathrm{r}$ & -0.086 & 0.146 & 0.123 & 0.07 \\
\hline & $\mathrm{p}$ value & 0.289 & 0.080 & 0.006 & 0.075 & & $\mathrm{p}$ value & 0.556 & 0.317 & 0.401 & 0.635 \\
\hline \multirow{2}{*}{ AoS } & $\mathrm{r}$ & -0.049 & -0.253 & 0.154 & 0.209 & \multirow{2}{*}{ FS } & $\mathrm{r}$ & -0.235 & 0.073 & 0.194 & 0.116 \\
\hline & $\mathrm{p}$ value & 0.738 & 0.079 & 0.290 & 0.15 & & $\mathrm{p}$ value & 0.104 & 0.618 & 0.181 & 0.428 \\
\hline \multirow{2}{*}{ AoD } & $\mathrm{r}$ & -0.439 & -0.517 & 0.475 & 0.520 & \multirow{2}{*}{ LVMI } & $\mathrm{r}$ & 0.087 & -0.212 & 0.02 & 0.075 \\
\hline & $\mathrm{p}$ value & 0.002 & $<0.001$ & 0.001 & $<0.001$ & & $\mathrm{p}$ value & 0.552 & 0.143 & 0.889 & 0.607 \\
\hline \multirow{2}{*}{ IVSD } & $\mathrm{r}$ & 0.026 & -0.246 & 0.085 & 0.152 & \multirow{2}{*}{$\mathrm{CHO}$} & $\mathrm{r}$ & 0.094 & 0.094 & -0.12 & -0.144 \\
\hline & $p$ value & 0.86 & 0.088 & 0.56 & 0.298 & & $\mathrm{p}$ value & 0.519 & 0.519 & 0.41 & 0.324 \\
\hline \multirow{2}{*}{ LVDD } & $\mathrm{r}$ & 0.064 & -0.134 & 0.049 & 0.075 & \multirow{2}{*}{ HDL } & $\mathrm{r}$ & -0.262 & -0.199 & 0.206 & 0.199 \\
\hline & $\mathrm{p}$ value & 0.661 & 0.358 & 0.736 & 0.611 & & $\mathrm{p}$ value & 0.068 & 0.171 & 0.155 & 0.169 \\
\hline \multirow[b]{2}{*}{ PWD } & $\mathrm{r}$ & 0.167 & -0.124 & -0.152 & -0.113 & \multirow[b]{2}{*}{ LDL } & $\mathrm{r}$ & 0.12 & -0.038 & -0.076 & -0.09 \\
\hline & $\mathrm{p}$ value & 0.252 & 0.396 & 0.297 & 0.440 & & $\mathrm{p}$ value & 0.412 & 0.793 & 0.606 & 0.537 \\
\hline \multirow{2}{*}{ IVSS } & $\mathrm{r}$ & 0.054 & -0.184 & -0.007 & 0.034 & \multirow{2}{*}{ TG } & $\mathrm{r}$ & 0.072 & 0.053 & -0.049 & -0.028 \\
\hline & $\mathrm{p}$ value & 0.712 & 0.205 & 0.964 & 0.816 & & $\mathrm{p}$ value & 0.621 & 0.717 & 0.739 & 0.849 \\
\hline \multirow{2}{*}{ LVDS } & $\mathrm{r}$ & 0.121 & -0.148 & -0.049 & -0.009 & & & & & & \\
\hline & $\mathrm{p}$ value & 0.406 & 0.310 & 0.739 & 0.952 & & & & & & \\
\hline
\end{tabular}




\section{DISCUSSION}

Development of early myocardial, coronary artery changes and many CVD complications are related to obesity in childhood and adolescence. To the best of our knowledge, the present study was the first to assess conventional echocardiography, arterial stiffness and lipid profiles in obese or overweight children. The study revealed that LVDS, LVDD, PWD, IVSS, LVDS, PWS, LVMI, AS, AD were higher in obese children when EF, FS, AS beta index and PSEM were lower compared to normal BMI children significantly. PWD had higher level in obese children compared with those overweight when, the parameters of arterial stiffness and lipid profiles were similar.

Zoair et al. ${ }^{15}$ conducted a study on obese children and found; EF, FS, LVMI and LVDD were higher in obesity when, LVMI and LVDD were significant. Vitarelli et al. ${ }^{16}$ led a study on hypercholesterolemia children aged 6-18 years consisted of normal weight and obese equally matched in sex and age with healthy children. They found higher PWD, IVSD, LVDD and LVMI in obese children with hypercholesterolemia compared to healthy ones. Few studies resulted that obesity has a strong impact on diastolic function in children. ${ }^{17}$

Amongst them, Ozdemir el al. ${ }^{17}$ compared obese and lean children and found; SBP, DBP, IVSD, PWD, IVSS, PWS, RWT and LVMI were higher in obese children significantly, when; LVDD, LVDS, EF and FS were higher but not significant. The findings of these studies were similar with present study results. They also found that children with hypercholesterolemia had an increase in systolic and diastolic BP but not significant, whereas we found a significant increase of systolic BP in obese children significantly.

Ghandi al al. ${ }^{18}$ reported that heart rate and systolic and diastolic BP were similar in obese and control children when resting heart rate and $\mathrm{BP}$ were significantly higher in obese children. They also found that the EF was similar but with normal range when LVMI, and LVDD were significantly greater in the obese similar with our findings.

Arterial stiffening is an ageing marker with atherosclerotic result and losing vessels flexibility affected by hypertension, chronic inflammatory and dyslipidemias. Arterial stiffening presented by AS, AD, AS beta index, PSEM and sometimes with pulse wave velocity (PWV) and augmentation index (AIx). Lentferink et al. ${ }^{3}$ considered PWV and Aix as measures of arterial stiffness and found that PWV was higher in obese children compared with lean children when Aix was similar. Kulsum-Mecci et al. ${ }^{4}$ found a significant increase of PWV in obese children. In this regards, the present study found that AS, AD were higher in obese or overweight children when AS beta index and PSEM were lower compared to controls. The differences were the type of AS parameters.

Similarly, Urbina et al. ${ }^{19}$ found that obese children had $20 \%$ more stiffing than those with normal weight. Hudson et al. ${ }^{20}$ indicated that obesity in children was associated with increasing arterial stiffening characterized by decreased distensibility when the present study found an increase of arterial distensiblity in obese or overweight children. The hypothesis for the illogical decrease likely is due to earlier puberty, peak arterial ability and increased body size in obese children. Jakab et al. ${ }^{21}$ directed an investigation on arterial stiffness in overweight and obese compared to normal children. They found that overweight and obese had same status of arterial stiffness compared with the peers similar with our findings. Pathophysiological outcomes in the circulatory framework because of weight are partially compensated hemodynamically that happened likely by the opening of small arterioles, by diminishing peripheral arteriolar resistance for this paradox. It isn't yet clear what factors, regardless of whether methodological, or qualities of the investigation, represent the distinctions among the examinations that have estimated the impact of stoutness on blood vessel consistence when as an end announced that fat youngsters don't have any immediate impact on the blood vessel capacities without solid proof.

Beside, has been demonstrated that stiffing had a strong correlation with early atherosclerosis disease in obese children. Obesity might also increase early atherosclerosis through a direct impact on vascular physiology. Such that more obesity in children influences on CVD level in adults. The results of the present study suggest that obesity has concurrent sign of increased stiffening, especial in central arteries. Lipid profiles related to abdominal fat, especially decreased HDL and increased $\mathrm{CHO}$, TG, and LDL are independent risk factors for CVD. ${ }^{15}$ Rizk et al. $^{22}$ considered waist circumference as a CVD risk factor and found that waist circumference had a positive association with lipids except HDL with negative association. In those who had high levels of TG, LDL and CHO the odds of CVD risk increased 6.3, 3.18 and 1.88 times more compared with those were normal. They also found that obesity is a risk of atherosclerosis by about two times more. Wang et al. $^{23}$ found the impact of lipid profiles on LVMI. Although in the present study, the obesity or overweight considered by BMI measurements, the same results observed such that the children who had abnormal TG and LDL had lower AOS and higher LVMI respectively. These results suggest that a favorable cardio metabolic risk may be retained later in life, independent of weight status during childhood.

Arjona-Villicaña et al. $^{24}$ conducted a study on obese children and found that lipid profiles had a correlation with cardiovascular risk factors when this correlation was positive for TG and LDL. The most common abnormal 
lipid profiles were low HDL plus hypertriglyceridemia. Shahid et al. ${ }^{25}$ proved that lipid profiles are important factor that contributes to the development of obesity and CHD. In obese children, although the levels of fat are high the correlations between the degree of obesity and lipid levels could help the pediatricians to predict dyslipidemia based on BMI. We demonstrated that from the conventional echocardiography parameters, AoS and LVMI changed in obese or overweight children when their lipid profiles were abnormal such that AoS was higher in obese children with $\mathrm{TG} \geq 150$ and LVMI was higher in obese children with LDL $<130$ significantly. Regarding arterial stiffing parameters, the results of the present study showed no changes. The aorta has dual functions: first, it acts as a conduit to transport blood throughout the body, and secondly, it acts as a cushion to accommodate ventricular ejection. The aorta can accommodate approximately $50 \%$ of blood volume ejected from the left ventricle. This elastic property of the aorta is important for optimal tissue perfusion and cardiovascular performance.

The characteristics of elastic in arteries are a cause of decrease in cardiac output, increases in perfusion of coronary artery, and is associated with reduced atherosclerotic progression.

Individuals with elevated aortic stiffness had a higher chance of experiencing a cardiovascular event after adjusting for age, sex, and standard risk factors. Aortic stiffing measures are more powerful marker compared to traditional variables such as blood pressure, lipid levels, or glucose levels since it reflects a long- term biophysical function of the arterial wall. These results provide knowledge about the importance of lipid profiles abnormality in children with overweight or obesity.

Because, from the study resulted that this abnormality has a significant effect only on LVMI and AoS. Our study emphasized the importance of measuring aortic stiffness during clinical evaluation of vascular functions specially those who are obese. Small sample size was the only study limitation such that with this insufficient samples, is hard to find the differences in the parameters of the study.

\section{CONCLUSION}

Heart functions were most at risk in obese children and obesity had a strong effect on aortic elasticity. Amongst obese or overweight children, PWD was higher in obese children, when all other heart functions were similar. From the study also concluded that in obese or overweight children, the variations of lipid profiles did not show the changes in heart functions except LVMI that was higher in children with abnormal LDL. Suggested for a study to recognize which factor of lipid profiles or obesity is more effected on heart damage functions. Based on the conclusion of the present study, since the aortic stiffing accelerated by obese, this group of the population should reduce obesity with specific programs.

\section{AUTHORS' CONTRIBUTION}

NMN: Concept and design, data acquisition, interpretation, drafting, final approval, and agree to be accountable for all aspects of the work. MNM, AT: Data acquisition, interpretation, drafting, final approval and agree to be accountable for all aspects of the work.

Conflict of interest: Authors declared no conflict of interest.

Acknowledgements: The authors would like to present their acknowledgments to the children's parents especially those who followed the visits foe a year after discharge from the hospitals.

\section{REFERENCES}

1. Azizian M, Mahdipour E, Mirhafez SR, Shoeibi S, Nematy M, Esmaily $\mathrm{H}$, et al. Cytokine profiles in overweight and obese subjects and normal weight individuals matched for age and gender. Ann Clin Biochem. 2016;53(6):663-8.

2. Heidari-Beni M, Kelishadi R. Prevalence of weight disorders in Iranian children and adolescents. Arch Iran Med. 2019 ;22(9):5115 .

3. Lentferink YE, Kromwijk LA, van der Aa MP, Knibbe CA, van der Vorst MM. Increased arterial stiffness in adolescents with obesity. Global Pediatr Health. 2019 b;6:2333794X19831297.

4. Kulsum- Mecci N, Goss C, Kozel BA, Garbutt JM, Schechtman $\mathrm{KB}$, Dharnidharka VR. Effects of obesity and hypertension on pulse wave velocity in children. J Clin Hypertens (Greenwich). 2017;19(3):221-6.

5. Cote AT, Harris KC, Panagiotopoulos C, Sandor GG, Devlin AM. Childhood obesity and cardiovascular dysfunction. J Am Coll Cardiol. 2013;62(15):1309-19.

6. Sethi S, Rivera O, Oliveros R, Chilton R. Aortic stiffness: pathophysiology, clinical implications, and approach to treatment. Integr Blood Press Control. 2014;7:29-34.

7. Mozos, I, Malainer, C, Horbańczuk, J. Inflammatory markers for arterial stiffness in cardiovascular diseases. Front Immunol. 2017;8:1058

8. Panchangam, C, Merrill, ED, Raghuveer, G. Utility of arterial stiffness assessment in children. Cardiol Young. 2018;28:362-376.

9. Snijder, MB, Stronks, K, Agyemang, C, Busschers, WB, Peters, $\mathrm{RJ}$, van den Born, BJ. Ethnic differences in arterial stiffness the Helius study. Int J Cardiol. 2015;191:28-33.

10. Csige I, Ujvárosy D, Szabó Z, Lőrincz I, Paragh G, Harangi M, Somodi S. The impact of obesity on the cardiovascular system. J Diab Res. 2018;2018:3407306.

11. Noori NM, Teimouri A, Keshavarz K, Moradi M. Assessment of aortic elasticity and the doppler tissue echocardiography in thalassemia major children. J Child Sci. 2020;10(01):e63-73.

12. Brady TM, Appel LJ, Holmes KW, Fivush B, Miller ER III. Association between adiposity and left ventricular mass in children with hypertension. J Clin Hypertens 2016; 18:625-33

13. Noori N, Nakhaee Moghadam M, Razzaghian Pour M, Teimouri A, Bagheri H, Yazdanparast A. Tissue Doppler Imaging versus Conventional Echocardiography in Evaluation of Cardiac Functions in Diabetes Mellitus. Int J Pediatr. 2019;7(7):9677-91.

14. Noori N, Nakhaee-Moghadam M, Teimouri A, Bagheri H. Tissue Doppler Imaging Findings and Lipid Profile Changes in Diabetes Mellitus Type I Children. Int J Pediatr. 2019;7(12):10423-39.

15. Zoair AM, Muhammad KT, Abu-Ammo DE, Motawea MM. Lipid profile and some cardiac functions in children with obesity. Gaz Egypt Paediatr Assoc . 2013;61(1):15-22. 
16. Vitarelli A, Martino F, Capotosto L, Martino E, Colantoni C, Ashurov R, et al. Early myocardial deformation changes in hypercholesterolemic and obese children and adolescents: a 2D and 3D speckle tracking echocardiography study. Medicine (Baltimore). 2014;93(12):e71

17. Ozdemir O, Hizli S, Abaci A, Agladioglu K, Aksoy S. Echocardiographic measurement of epicardial adipose tissue in obese children. Pediatr Cardiol. 2010;31(6):853-60.

18. Ghandi Y, Sharifi M, Habibi D, Dorreh F, Hashemi M. Evaluation of left ventricular function in obese children without hypertension by a tissue Doppler imaging study. Ann Pediatr Cardiol. 2018;11(1):28.

19. Urbina EM, Khoury PR, McCoy CE, et al. Triglyceride to HDL$\mathrm{C}$ ratio and increased arterial stiffness in children, adolescents, and young adults. Pediatrics. 2013;131:e1082- e1090.

20. Hudson LD, Rapala A, Khan T, Williams B, Viner RM. Evidence for contemporary arterial stiffening in obese children and adolescents using pulse wave velocity: A systematic review and meta-analysis. Atherosclerosis. 2015;241(2):376-86.
21. Jakab AE, Hidvégi EV, Illyés M, Cziráki A, Kalmár T, Maróti Z, et al. Childhood Obesity: Does it Have Any Effect on Young Arteries?. Front Pediatr. 2020;8:389.

22. Rizk NM, Yousef M. Association of lipid profile and waist circumference as cardiovascular risk factors for overweight and obesity among school children in Qatar. Diabetes, Metab Synd Obes: 2012;5:425-32.

23. Wang H, Li Z, Guo X, Chen Y, Chang Y, Chen S, et al. The impact of nontraditional lipid profiles on left ventricular geometric abnormalities in general Chinese population. BMC Cardiovasc Disord. 2018;18(1):1-1.

24. Arjona-Villicaña RD, Herrera-Sánchez LF, Sumárraga-Ugalde $\mathrm{CD}$, Alcocer-Gamboa MA. Relationship between body mass index and lipid profile in obese mexican children and adolescents: A retrospective analysis. BoletínMédico Del Hospital Infantil de México. 2014;71(2):88-94.

25. Shahid SU, Sarwar S. The abnormal lipid profile in obesity and coronary heart disease (CHD) in Pakistani subjects. Lipids Health Dis. 2020;19:1-7.

\section{Address for Correspondence:}

Dr. Alireza Teimouri, Children \& Adolescent Health Research Center, Resistant Tuberculosis Institute, School of Medicine, Zahedan Univ Med Sci, Zahedan, Iran.

Email: alirezateimouri260@gmail.com 